Article

\title{
Renormalizable Gravitational Action That Reduces to General Relativity on the Mass-Shell
}

\author{
Peter D. Morley
}

System of Systems Analytics, Inc., 11250 Waples Mill Road, Suite 300, Fairfax, VA 22030-7400, USA;

peter3@uchicago.edu

Received: 10 July 2018; Accepted: 27 July 2018; Published: 30 July 2018

check for updates

\begin{abstract}
We derive the equation that relates gravity to quantum mechanics: $\left.R\right|_{\text {mass }- \text { shell }}=\frac{8 \pi G}{c^{4}} L_{S M}$, where $R$ is the scalar curvature, $G$ is the gravitational constant, $c$ is the speed of light and $L_{S M}$ is the Standard Model Lagrangian, or its future replacement. Implications of this equation are discussed in the paper. In particular, we show (in the last section) that this equation is the transformation that relates four-dimensional physics to two-dimensional physics.
\end{abstract}

Keywords: scalar curvature; renormalizability

\section{Introduction}

General Relativity (GR) is described by the following combined action ${ }^{1}$, called the Einstein-Hilbert action, $\mathcal{S}_{\mathcal{E}}$, where we link the Lagrangian of the Standard Model of particle physics $\left(L_{S M}=S U(3)_{C} \times\right.$ $\left.S U(2)_{L} \times U(1)_{Y}\right)$ to GR (using the $(-+++)$ metric signature, with $\left.g=\operatorname{det}\left[g_{\mu \nu}\right]\right)$ :

$$
\mathcal{S}_{\mathcal{E}}=\int\left\{\frac{1}{2 \kappa} R+L_{S M}\right\} \sqrt{-g} d^{3} x d t
$$

In Equation (1), the constant $\kappa=\frac{8 \pi G}{c^{4}}$. This action is non-renormalizable [1,2] in four-dimensional space-time. For the purposes of this paper then, we discard $\mathcal{S}_{\mathcal{E}}$ and replace it with a new action $\mathcal{S}_{\mathcal{N} \mathcal{P}}$. In considering a replacement for $\mathcal{S}_{\mathcal{E}}$, we require that the classical General Relativity equations of motion are maintained; only the off-mass-shell behavior can be modified. This requires that the high energy momentum components from the gravity Lagrangian be damped. Wilson [3] has shown us how to do that by introducing a smooth cutoff function $\mathcal{K}$ in the denominator of the term in which we are interested. This program was carried through by Igarashi, Itoh and Sonoda [4], who showed that different choices of the smooth cutoff function merely amount to reparameterization and renormalization of the fields.

The only $\mathcal{K}$ available is $L_{S M}$, so the ratio $R / L_{S M}$ must be present. Finally, this ratio cannot appear in a polynomial, which would vanish when gravity is neglected, thereby giving the exponential form:

$$
\mathcal{S}_{\mathcal{N P}}=\int e^{\frac{R}{2 Q L_{S M}}} L_{S M} \sqrt{-g} d^{3} x d t
$$

where $Q$ is a constant and $Q \neq \kappa$ (shown below). The subscript 'NP' designates 'non-polynomial'. The replacement $\mathcal{S}_{\mathcal{E}} \rightarrow \mathcal{S}_{\mathcal{N} \mathcal{P}}$ has profound implications for physics, which this paper discusses.

Donoghue [5] describes the present arguments that General Relativity is a perfectly acceptable effective field theory. Understanding this to mean that low energy gravity must be renormalizable,

1 We discuss the cosmological constant in Section 3.1 below. 
the search is then on to find the coupled-matter-gravity action that replaces $\mathcal{S}_{\mathcal{E}}$. That this approach could lead to valuable results is emphasized by the work of Halpern and Huang [6], who showed that non-polynomial Lagrangians have surprising new physics.

\section{Equations of Motion with Noether's Theorem}

The coupling of gravity and matter in $\mathcal{S}_{\mathcal{N P}}$ prohibits the ability to add four-vector divergences to either gravity or matter, because the divergence theorem is no longer applicable. The action is stationary for fields that are solutions of the actual equations of motion; in other words, the variation is over those fields that satisfy the symmetries of the problem. This allows non-polynomial Lagrangians, if desired ${ }^{2}$. The extremum condition is:

$$
\begin{aligned}
\delta \mathcal{S}_{\mathcal{N P}}=0 & =\int\left\{\frac{\delta}{\delta g^{\mu \nu}}\left[e^{\frac{R}{2 Q L_{S M}}}\right] L_{S M} \sqrt{-g}\right. \\
& \left.+\frac{\delta}{\delta g^{\mu \nu}}\left[L_{S M} \sqrt{-g}\right] e^{\frac{R}{2 Q L_{S M}}} L_{S M}\right\} \delta g^{\mu v} d^{3} x d t \\
& +\int\left\{\sum _ { i } \left[e^{\frac{R}{2 Q L_{S M}}} L_{S M} \sqrt{-g} \delta_{i}\left(\frac{R}{2 Q L_{S M}}\right)\right.\right. \\
& \left.\left.+e^{\frac{R}{2 Q L_{S M}}} \sqrt{-g} \delta_{i}\left(L_{S M}\right)\right]\right\} d^{3} x d t
\end{aligned}
$$

In Equation (3), $\sum_{i}$ denotes the variation $\delta_{i}$ over the fields in $L_{S M}$. Note that $\sqrt{-g}$ and $R$ do not involve $e^{3}$ the variation associated with $\delta_{i}$. The individual variations are independent of each other and separately are extrema.

\subsection{Gravitational Equations of Motion}

The gravitational equations of motion are determined by:

$$
0=\int\left\{\frac{\delta}{\delta g^{\mu \nu}}\left[e^{\frac{R}{2 Q L_{S M}}}\right] L_{S M} \sqrt{-g}+\frac{\delta}{\delta g^{\mu \nu}}\left[L_{S M} \sqrt{-g}\right] e^{\frac{R}{2 Q L_{S M}}} L_{S M}\right\} \delta g^{\mu v} d^{3} x d t
$$

In contemplating what this may reduce to, we consider the fact that $\mathcal{S}_{\mathcal{E}}$, as a classical field theory, satisfies all macroscopic experimental data tests. Thus, we would expect that $\mathcal{S}_{\mathcal{N} \mathcal{P}}$ has the same classical behavior as $\mathcal{S}_{\mathcal{E}}$, but different quantum behavior. In particular, $\mathcal{S}_{\mathcal{N} \mathcal{P}}$ can be a renormalizable action that will be discussed in Section 3. Equation (4) reduces to:

$$
0=\int d^{3} x d t e^{\frac{R}{2 Q L_{S M}}}\left\{\frac{\sqrt{-g}}{2 Q} \frac{\delta R}{\delta g^{\mu v}}+\frac{\delta L_{S M}}{\delta g^{\mu \nu}} \sqrt{-g}\left(1-\frac{R}{2 Q L_{S M}}\right)+L_{S M} \frac{\delta(\sqrt{-g})}{\delta g^{\mu \nu}}\right\} \delta g^{\mu \nu}
$$

We do each term separately.

$$
\delta R=R_{\mu v} \delta g^{\mu v}+g^{\mu v} \delta R_{\mu v}
$$

However, the variations must satisfy the symmetry properties of GR, which is ${ }^{4} g^{\mu v} \delta R_{\mu v}=0$. Therefore:

$$
\frac{\delta R}{\delta g^{\mu \nu}}=R_{\mu v}
$$

\footnotetext{
No integration by parts is required.

This is true even though the gravitational field has matter fields as sources.

This is a four-vector divergence (see for example [7]), which is zero by Noether's principle.
} 
Working out the remaining terms:

$$
\frac{1}{\sqrt{-g}} \frac{\delta \sqrt{-g}}{\delta g^{\mu \nu}}=-\frac{1}{2} g_{\mu \nu}
$$

and:

$$
\frac{\delta L_{S M}}{\delta g^{\mu \nu}}=-\frac{1}{2}\left(T_{\mu \nu}-g_{\mu \nu} L_{S M}\right)
$$

In Equation (9), $T_{\mu \nu}$ is the stress-energy tensor. Putting these altogether gives:

$$
0=\int d^{3} x d t e^{\frac{R}{2 Q L_{S M}}}\left[\frac{\sqrt{-g}}{2 Q} R_{\mu \nu}+\frac{\sqrt{-g}}{2}\left(g_{\mu \nu} L_{S M}-T_{\mu v}\right)\left(1-\frac{R}{2 Q L_{S M}}\right)+L_{s m}\left(\frac{-\sqrt{-g}}{2} g_{\mu \nu}\right)\right] \delta g^{\mu \nu}
$$

The arbitrary variation giving zero requires that the complete term in parenthesis must be zero, producing:

$$
R_{\mu \nu}-\frac{R}{2} g_{\mu \nu}=\left(Q-\frac{\left.R\right|_{\text {mass-shell }}}{2 L_{S M}}\right) T_{\mu \nu}
$$

Now, the conservation of energy-momentum from the tensor $T_{\mu \nu}$ requires:

$$
\frac{\left.R\right|_{\text {mass-shell }}}{2 L_{S M}}=\text { constant } \equiv \zeta
$$

We now can evaluate the two constants $Q, \zeta$ using the technique in [8], by requiring that the gravitational interaction reduces to the static Newtonian potential. Operating on Equation (11) by $g^{\mu \nu}$ gives:

$$
R-\frac{R}{2}(4)=\left(Q-\frac{\left.R\right|_{\text {mass-shell }}}{2 L_{S M}}\right) T
$$

where $^{5} T=-L_{S M} \rightarrow$ energy density. Noting that all the $R$ in Equation (13) are on the mass-shell (equation of motion), we momentarily drop the designation and have:

$$
-R=Q T+\frac{R}{2} \text { or }-\frac{3 R}{2}=Q T
$$

In order to satisfy Newton's law of gravitation, the equation for the scalar curvature must reduce, in that particular case, to $R=-8 \frac{\pi G}{c^{4}} T$, so the constants are $Q=12 \frac{\pi G}{c^{4}}$ and $\zeta=4 \frac{\pi G}{c^{4}}$. Thus, the central equations for the non-polynomial action are:

$$
\left.R\right|_{\text {mass-shell }}=\frac{8 \pi G}{c^{4}} L_{S M}
$$

with the equation of motion:

$$
R_{\mu v}-\frac{R}{2} g_{\mu v}=\frac{8 \pi G}{c^{4}} T_{\mu v}
$$

By using the original Einstein-Hilbert action, Equation (15) has gone missing. We note here that the scalar curvature is already known to be non-vanishing only within matter, so Equation (15) would not be an unexpected finding ${ }^{6}$.

5 In the static limit.

6 The scalar curvature can be positive or negative, consistent with Equation (15). 


\subsection{Matter Equations of Motion}

The other extremum from Equation (3) is:

$$
0=\int\left\{\sum_{i}\left[e^{\frac{R}{2 Q L_{S M}}} L_{S M} \sqrt{-g} \delta_{i}\left(\frac{R}{2 Q L_{S M}}\right)+e^{\frac{R}{2 Q L_{S M}}} \sqrt{-g} \delta_{i}\left(L_{S M}\right)\right]\right\} d^{3} x d t
$$

We work this out for a scalar field $\phi$ to demonstrate that the non-polynomial action is a viable action due to the symmetry properties of the variations, which gives rise to a zero four-vector divergence (Noether's theorem). Equation (17) becomes (for a single field):

$$
0=\int e^{\frac{R}{2 Q L_{S M}}} \sqrt{-g}\left(1-\frac{R}{2 Q L_{S M}}\right) \delta L_{S M} d^{3} x d t
$$

From the previous section:

$$
\left(1-\frac{R}{2 Q L_{S M}}\right)=\frac{2}{3}
$$

The term $\delta L_{S M}$ for a single scalar field $\phi$ is:

$$
\delta L_{S M}=\frac{\partial L_{S M}}{\partial \phi}(\Delta \phi)+\left(\frac{\partial L_{S M}}{\partial\left(\partial_{\mu} \phi\right)}\right) \partial_{\mu}(\Delta \phi)
$$

We use the identity:

$$
\partial_{\mu}\left\{\frac{\partial L_{S M}}{\partial\left(\partial_{\mu} \phi\right)} \Delta \phi\right\}=\Delta \phi \partial_{\mu}\left\{\frac{\partial L_{S M}}{\partial\left(\partial_{\mu} \phi\right)}\right\}+\frac{\partial L_{S M}}{\partial\left(\partial_{\mu} \phi\right)} \partial_{\mu}(\Delta \phi)
$$

Equation (20) becomes:

$$
\delta L_{S M}=\left\{\frac{\partial L_{S M}}{\partial \phi}-\partial_{\mu} \frac{\partial L_{S M}}{\partial\left(\partial_{\mu} \phi\right)}\right\} \Delta \phi+\partial_{\mu}\left\{\frac{\partial L_{S M}}{\partial\left(\partial_{\mu} \phi\right)} \Delta \phi\right\}
$$

However, the variations satisfy the symmetries of the Lagrangian and:

$$
\partial_{\mu}\left\{\frac{\partial L_{S M}}{\partial\left(\partial_{\mu} \phi\right)} \Delta \phi\right\}=0
$$

without requiring integration by parts or the divergence theorem (Noether's principle again). Because the integrand in Equation (18) involves a never-zero exponential ${ }^{7}$ and a $\frac{2}{3}$ factor, the terms multiplying $\Delta \phi$ must vanish, giving the equation of motion:

$$
0=\frac{\partial L_{S M}}{\partial \phi}-\partial_{\mu} \frac{\partial L_{S M}}{\partial\left(\partial_{\mu} \phi\right)}
$$

\section{Renormalizability of $\mathcal{S}_{\mathcal{N P}}$}

Before calculations are presented, we want to explain in an intuitive manner why $\mathcal{S}_{\mathcal{N} \mathcal{P}}$ and $\mathcal{S}_{\mathcal{E}}$ should differ when quantum fluctuations are taken into account. Firstly, Equation (15) shows that the scalar curvature is renormalized using the theorem in [9], which was derived in finite-temperature field theory for the thermodynamic potential $\Omega$. Secondly, the deleterious effects of having a coupling constant $G$ with units is ameliorated by $L_{S M}$ in the denominator of the exponential of $\mathcal{S}_{\mathcal{N P}}$. Radiative corrections will give rise to new, additional operators in the Hamiltonian: if their

7 This is critical for both Equations (10) and (18). 
coefficients $^{8}$ are finite, we say we are dealing with a renormalizable action; if the coefficients are infinite, the action becomes non-renormalizable. Thus, the statement that $\mathcal{S}_{\mathcal{N} \mathcal{P}}$ is renormalizable means that all the radiative corrections are finite after normal mass, charge and wavefunction renormalizations are performed. We now go into the details.

\subsection{Renormalization of the Scalar Curvature $R$}

The inputs to a gravitational calculation are the stress-energy tensor, $T_{\mu v}$, with boundary conditions. The Lagrangian in Equation (15) is used to calculate the components of $T_{\mu \nu}$, and Equation (16) gives the solution for the Einstein tensor. $R$ in finite-temperature quantum field theory is given by vacuum diagrams. The thermodynamic potential $\Omega$ in finite-temperature quantum field theory is renormalized by the theorem ${ }^{9}$ of [9]:

$$
\Omega_{B}\left(\left\{g_{B i}\right\},\left\{m_{B i}\right\}, T,\left\{\mu_{i}\right\}\right)-\Omega_{B}\left(\left\{g_{B i}\right\},\left\{m_{B i}\right\}, T=0,\left\{\mu_{i}=0\right\}\right)=\Omega_{R}\left(\left\{g_{R i}\right\},\left\{m_{R i}\right\}, T,\left\{\mu_{i}\right\}\right)
$$

where $B$ and $R$ refer to bare and renormalized quantities, $\left\{g_{i}\right\},\left\{m_{i}\right\}$ refer to the collection of coupling constants and masses and $T,\left\{\mu_{i}\right\}$ refer to temperature and the various chemical potentials. When quantum corrections are derived for $R$, the renormalized ${ }^{10}$ scalar curvature $R_{R}$ is:

$$
R_{B}\left(\left\{g_{B i}\right\},\left\{m_{B i}\right\}, T,\left\{\mu_{i}\right\}\right)-R_{B}\left(\left\{g_{B i}\right\},\left\{m_{B i}\right\}, T=0,\left\{\mu_{i}=0\right\}\right)=R_{R}\left(\left\{g_{R i}\right\},\left\{m_{R i}\right\}, T,\left\{\mu_{i}\right\}\right)
$$

From Equation (26), we see immediately that the various condensates (Higgs, chiral, etc.) associated with phase transitions do not contribute to $R_{R}{ }^{11}$.

The finite and tiny cosmological constant $\lambda_{c o s} \neq 0$ has an unknown origin at the present time. To illustrate Equation (15), we give a toy model for $\lambda_{c o s}$ : a condensation of a sterile neutrino of mass $m_{\text {toy }}$ that fills up positive energy levels in the Universe to Fermi momentum $p_{\text {toy. The Fermi momentum }}$ replaces the chemical potential $\mu$ in Equation (26). From Equation (15):

$$
\begin{aligned}
<R> & =\frac{8 \pi G}{c^{4}}<L_{S M}> \\
& =\frac{8 \pi G}{c^{2}} \rho_{t o y}
\end{aligned}
$$

where $\rho_{t o y}$ is the mass density. The mass density of such a neutrino is given by Equation (10) in [10].

$$
\rho_{t o y}=\frac{\pi m_{t o y}^{4} c^{3}}{h^{3}}\left\{2 x\left(1+x^{2}\right)^{3 / 2}-x \sqrt{1+x^{2}}-\sinh ^{-1} x\right\}
$$

where $x=\frac{p_{t o y}}{m_{\text {toy }} c}$.

The relationship between $\lambda_{\cos }$ and $\langle R>$ is:

$$
<R>=4 \lambda_{\cos }
$$

Then, for this toy model:

$$
\lambda_{c o s}=\frac{2 \pi^{2} G m_{t o y}^{4} c}{h^{3}}\left\{2 x\left(1+x^{2}\right)^{3 / 2}-x \sqrt{1+x^{2}}-\sinh ^{-1} x\right\}
$$

\footnotetext{
These being after having done the mass, charge and wavefunction renormalizations.

Here, this is written for on-mass-shell renormalization, so no Euclidean subtraction momentum squares appear.

The set of coupling constants includes Newton's constant $G$.

11 This is because these condensates are present under the conditions $T=0,\left\{\mu_{i}=0\right\}$ and so cancel from the two terms on the left-hand side of Equation (26).
} 
A value of $\lambda_{\cos }=1.11 \times 10^{52} / \mathrm{m}^{2}$ is an approximation, [11]. If we arbitrarily assign a value of $m_{\text {toy }} c^{2}=10 \mathrm{meV}(0.01 \mathrm{eV})$, then $x=0.647 \ldots$

\subsection{Finite Radiative Corrections to $\mathcal{S}_{\mathcal{N P}}$}

We do background gauge field quantization: by expanding locally, the short distance behavior is always flat space for a smooth manifold. Following [1], $g_{\mu v}=\eta_{\mu v}+h_{\mu v}$ leads to $\sqrt{-g} R \sim(\partial h)^{2}+$ $(\partial h)^{2} h+\cdots$ and in Equation (2), letting $h=\sqrt{Q} \tilde{h}$ :

$$
e^{\frac{R}{2 L_{S M}}} \sim e^{\frac{1}{2 L_{S M} \sqrt{-g}}\left[(\partial \tilde{h})^{2}+\sqrt{Q}(\partial \tilde{h})^{2} \tilde{h}+\cdots\right]}
$$

The $L_{S M}$ term in Equation (31) acts like a smooth momentum cutoff function, as long as $L_{S M}$ itself is renormalizable ${ }^{12}$. Thus, the matter Lagrangian $L_{S M}$ plays a critical role in the renormalization of gravity. This cutoff behavior results in an effective re-scaling of the metric:

$$
e^{\frac{R}{2 Q L_{S M}}} \sqrt{-g} \equiv \sqrt{-g^{\prime}}
$$

where $\sqrt{-g^{\prime}}$ is a finite quantity, after the standard mass, coupling constant and wavefunction renormalizations are performed.

\section{General Implications of $\mathcal{S}_{\mathcal{N} \mathcal{P}}$}

\subsection{Comment on the Black Hole Information Paradox}

The black hole information paradox refers to the Hawking radiation being emitted by a black hole $^{13}$. If the photon radiation is truly thermal, it cannot carry away information ${ }^{14}$. The radiation need not be photons. A positron-electron pair can be created outside a black hole, and one Fermion traverses the event horizon, while the other goes to infinity. The black hole has then "eaten up" information because ostensibly the scalar curvature $R$ just records an energy density/angular momentum change, which could have been any particles. However, Equation (15) changes this situation. Instead of an energy density change, the Lagrangian density changes. This quantity carries with it all the symmetries of matter, including the Fermion number. Equation (15) is a ledger inside the black hole, even if no experiment can do an audit. A detailed calculation has to be implemented on the eventual end process of black hole evaporation, but the back-reaction is now just an elementary particle recoil, with all symmetries of $L_{S M}$ having to be conserved.

Other authors $[13,14]$ have already concluded that there is no information loss from black hole evaporation because black holes have surface normal modes that give rise to pure quantum states, not mixed thermal states.

\subsection{Implications for the Matter Lagrangian $L_{S M}$}

The QCD (Quantum Chromodynamics) Lagrangian $L_{S U(3)}$ piece of $L_{S M}$ has a possible effective Lagrangian $L_{S U(3)} f f$, which is:

$$
L_{S U(3)_{e} f f}=L_{S U(3)}+\frac{\theta}{16 \pi^{2}} \operatorname{tr}\left(F_{\mu \nu} \tilde{F}^{\mu v}\right)
$$

\footnotetext{
12 As shown in [1], $\partial_{t} L_{S M}$ appears in the numerator of the renormalization group flow for changes of renormalization scales, where $t$ is a renormalization 'time' $t=\ln \frac{\Lambda_{0}}{\Lambda}$ and $t \rightarrow \infty$ as $\Lambda$ decreases.

13 For a review, see [12].

14 For example, it cannot radiate polarized light.
} 
where $F_{\mu \nu}^{a}$ and $\tilde{F}^{b \mu v}$ are the familiar gluon color matrix tensor and color dual-field matrix tensor, respectively, and $\operatorname{tr}$ means the trace in color space. The extra piece $\frac{\theta}{16 \pi^{2}} \operatorname{tr}\left(F_{\mu \nu} \tilde{F}^{\mu v}\right)$ is the four-divergence of a vector. If $\mathcal{S}_{\mathcal{E}}$ is being used, the Divergence Theorem can be invoked, and then, non-zero surface terms from instantons can lead to this term contributing to quantum processes. If instead, $\mathcal{S}_{\mathcal{N} \mathcal{P}}$ is used, then the extra $\theta$-piece stands on its own depending on experimental data determining the value of the $\mathrm{CP}$ (charge conjugation $x$ Parity) -violating parameter $\theta$. Using $\mathcal{S}_{\mathcal{N} \mathcal{P}}$ means just another parameter $\theta$, but no $\theta$-vacuum, since instantons are no longer relevant in the presence of this gravity-matter coupling.

An interesting possibility is the following: starting with $L_{S U(3)_{e} f f}$, compute the $\theta$ radiative corrections for the confining QCD ground state to see if $\theta=0$ is the minimum. This would be the normal QCD ground state that conserves $C P$. However, the excited ground state of freed quarks may have a different minimum $\theta \neq 0$. The latter calculation can be done in a perturbative manner. This is the suggestion put forward by [15] to explain CP violations in the early Universe.

\subsection{Reduction of Quantum Mechanical Probability Amplitudes}

Feynman [16] shows that the quantum mechanical amplitude is the sum over all paths, where each path has a phase proportional to the action $S$ :

$$
\phi=\text { const } \times e^{\frac{i}{\hbar} \int d^{3} x d t e^{\frac{R}{2 Q L_{S M}}} L_{S M} \sqrt{-g}}
$$

where const is a constant. Using Equation (15), the dominant contribution to $\phi$ can be evaluated directly. With $x^{0}=c t, c d^{3} x d t=d^{4} x$, and the definition of the Planck length $l_{P}$ :

$$
l_{P} \equiv \sqrt{\frac{\hbar G}{c^{3}}}
$$

Equation (34) reduces to:

$$
\phi \rightarrow e^{\frac{i \sqrt[3]{e}}{8 \pi} \int d^{4} x \frac{\left.R\right|_{\text {mass }- \text { shell }} \sqrt{-g}}{l_{P}^{2}}}
$$

Now, $\left.R\right|_{\text {mass }- \text { shell }} / l_{P}^{2}$ is the scalar curvature per unit area, and we conclude that the probability amplitude is the integration over space-time of the scalar curvature per unit area. The inescapable conclusion is that quantum mechanical information resides on two-dimensional surfaces. This is a concrete realization of the 'holographic principle' [17].

\section{Conclusions}

This paper discards the historical Einstein-Hilbert action and replaces it with a coupled gravity-matter non-polynomial interaction. The renormalizability of the scalar curvature follows the same mathematics as the renormalizability of the thermodynamic potential in finite-temperature quantum field theory. Radiative corrections to $\mathcal{S}_{\mathcal{N} \mathcal{P}}$ are finite due to the high momentum cutoff from the renormalized matter fields in $L_{S M}$. This leads to an effective finite metric tensor. Many details still remain to be worked out, including the calculation of the Hawking radiation, with the constraint now imposed by Equation (15).

Funding: This research received no external funding.

Conflicts of Interest: The author declares no conflict of interest.

\section{References}

1. Shomer, A. A pedagogical explanation for the non-renormalizability of gravity. arXiv 2007, arXiv:0709.35555v2.

2. Hooft, G.; Veltman, M. One loop divergences in the theory of gravitation. Ann. Henri Poincaré 1974, 20, 69-94.

3. Wilson, K.G.; Kogut, J.B. The renormalization group and the $\epsilon$ expansion. Phys. Rep. 1974, 12, 75-199. [CrossRef] 
4. Igarashi, Y.; Itoh, K.; Sonoda, H. Realization of Symmetry in the ERG approach to quantum field theory. Prog. Theor. Phys. Suppl. 2009, 181, 1-166. [CrossRef]

5. Donoghue, J.F. Introduction to the effective field theory description of gravity. In Advanced School on Effective Field Theories; Cornet, F., Herrero, M.J., Eds.; World Scientific: Singapore, 1997; Volume 26, pp. 217-224.

6. Halpern, K.; Huang, K. Fixed point structure of scalar fields. Phys. Rev. Lett. 1995, 74, 3526. [CrossRef] [PubMed]

7. Einstein-Hilbert Action. Available online: https://en.wikipedia.org/wiki/Einstein $\%$ E2\%80\%93Hilbert_ action (accessed on 26 July 2018).

8. Loveridge, L.C. Physical and geometric interpretations of the Riemann tensor, Ricci tensor and scalar curvature. arXiv 2004, arXiv:gr-qc/040199v1.

9. Morley, P.D. Thermodynamic potential in quantum electrodynamics. Phys. Rev. 1978, 17, 598. [CrossRef]

10. Morley, P.D.; Buettner, D.J. SHM of galaxies embedded within condensed neutrino matter. Int. J. Phys. D 2015, 24, 1550004. [CrossRef]

11. Cosmological Constant. Available online: https://en.wikipedia.org/wiki/Cosmological_constant (accessed on 26 July 2018).

12. Page, D.N. Hawking radiation and black hole thermodynamics. New J. Phys. 2005, 7, 203. [CrossRef]

13. Corda, C. Time-dependent Schrodunger equation for black hole evaporation: no information loss. Ann. Phys. 2015, 353, 71-82. [CrossRef]

14. Corda, C. Precise model of Hawking radiation from tunnelling mechanism. Classical Quant. Grav. 2015, 32, 195007. [CrossRef]

15. Morley, P.D.; Schmidt, I.A. Strong P, CP, T violations in heavy-ion collisions. Z. Phys. C Part. Fields 1985, 26, 627-628. [CrossRef]

16. Feynman, R.P.; Hibbs, A.R. Quantum Mechanics and Path Integrals; McGraw-Hill, Inc.: New York, NY, USA, 1965.

17. Bousso, R. The holographical principle. Rev. Mod. Phys. 2002, 74, 825. [CrossRef]

(c) 2018 by the author. Licensee MDPI, Basel, Switzerland. This article is an open access article distributed under the terms and conditions of the Creative Commons Attribution (CC BY) license (http:/ / creativecommons.org/licenses/by/4.0/). 